\title{
Qualitative and Quantitative Phytochemical Analysis of Ononis Hairy Root Cultures
}

\author{
Nóra Gampe', Zoltán Szakács², András Darcsi' ${ }^{1}$ Imre Boldizsár , Éva Szöke', \\ Inna Kuzovkina ${ }^{4}$, László Kursinszki1 and Szabolcs Béni ${ }^{1 *}$
}

\begin{abstract}
${ }^{1}$ Department of Pharmacognosy, Semmelweis University, Budapest, Hungary, ${ }^{2}$ Spectroscopic Research Department, Gedeon Richter Plc., Budapest, Hungary, ${ }^{3}$ Natural Bioactive Compounds Group, Institutional Excellence Program, Department of Plant Anatomy, Eötvös Loránd University, Budapest, Hungary, ${ }^{4}$ Timiryazev Institute of Plant Physiology, Russian Academy of Sciences, Moscow, Russia
\end{abstract}

\section{OPEN ACCESS}

Edited by:

Aymerick Eudes,

Lawrence Berkeley National Laboratory, United States

Reviewed by:

Edward Emmanuel Kweku Baidoo,

Particle Data Group, United States

Dejan S. Stojkovic,

University of Belgrade, Serbia

${ }^{*}$ Correspondence:

Szabolcs Bén

beni.szabolcs@

pharma.semme/weis-univ.hu;

beni.szabi@gmail.com

Specialty section:

This article was submitted to

Plant Metabolism

and Chemodiversity,

a section of the journal

Frontiers in Plant Science

Received: 28 October 2020 Accepted: 30 November 2020

Published: 13 January 2021

Citation:

Gampe N, Szakács Z, Darcsi A, Boldizsár I, Szőke É, Kuzovkina I,

Kursinszki L and Béni S (2021)

Qualitative and Quantitative Phytochemical Analysis of Ononis

Hairy Root Cultures.

Front. Plant Sci. 11:622585. doi: 10.3389/fpls.2020.622585
Hairy root cultures are genetically and biochemically stable, and they regularly possess the same or better biosynthetic capabilities for specialized (secondary) metabolite production compared to the intact plant. Ononis species are well-known herbal remedies in ethnopharmacology and rich sources of isoflavonoids. Besides isoflavones, less prevalent isoflavones and pterocarpans with valuable biological effects can be found in Ononis species as well. As these plants are only collected but not cultivated, biotechnological methods could play a role in the larger-scale extraction of Ononis isoflavonoids. Regarding this information, we aimed to establish Ononis spinosa and Ononis arvensis hairy root cultures (HRCs) and analyze the isoflavonoid profile of hairy root cultures qualitatively and quantitatively, in order to define their capacity to produce biologically valuable isoflavonoids. During the qualitative description, beside isoflavonoids, two new phenolic lactones, namely, bulatlactone 2 " $-O-\beta$-D-glucoside and ononilactone, were isolated, and their structures were characterized for the first time. Altogether, 29 compounds were identified by the means of UPLC-Orbitrap-MS/MS. Based on UHPLC-UV-DAD measurements, the isoflavonoid spectrum of the Ononis HRCs differed markedly from wild-grown samples, as they produce a limited range of the scaffolds. The most abundant compounds in the HRCs were medicarpin glucoside and sativanone glucoside. The overall isoflavonoid production of the cultures was comparable to wild-grown $\mathrm{O}$. arvensis and approximately twice as high as in wildgrown $O$. spinosa samples. As the overall content of wild-grown samples include more isoflavonoid derivatives, the HRCs contain structurally less divergent isoflavonoids but in higher quantity.

Keywords: Ononis, hairy root, biotechnology, isoflavonoid, bulatlactone, ononilactone

\section{INTRODUCTION}

Bioactive natural products are molecules perfected by evolution, which, based on their physicochemical properties, are much more likely to become potential drug candidates than synthetic compounds produced by combinatorial chemical methods (Larsson et al., 2005). Their sources might be herbs that are easy to cultivate, e.g., lavender or chamomile, etc., but in many cases, it 
is only possible to isolate it from wild-growing populations, e.g., taxol. This poses a major threat to ecological diversity, habitat undisturbedness, or even the survival of the species. An additional difficulty could be if the intact plant contains the active compound only as a minor component, and its extraction is not economical (Atanasov et al., 2015).

In vitro cultivation of medicinal plants can provide a solution to this problem. Micro-propagation, organ, and cell cultures present an opportunity to produce the desired species under laboratory conditions and to bioreplicate individuals and/or organs with the highest active substance content. Although plant cell cultures seemed to be very promising tools in the production of specialized metabolites, unfortunately, they often did not live up to their expectations. The reasons can lie in the difficulty of industrial upscaling. Another problem is that the lack of differentiation and compartmentalization, that can result in different metabolic profiles compared to the intact plant (Atanasov et al., 2015). Hairy root cultures, as transgenic tissue cultures, show a higher degree of differentiation. These are created by the bacterial infection of Agrobacterium rhizogenes, during which bacterial plasmid is incorporated into the plant DNA causing prolific growth of neoplastic roots. Hairy root systems can be maintained without the use of phytohormones. As opposed to cell cultures, they are genetically and biochemically stable, and they regularly possess the same or better biosynthetic capabilities for specialized metabolite production as the intact plant (Georgiev et al., 2012).

Isoflavonoids are products of the phenylpropanoid biosynthesis route, and their main groups are isoflavones, isoflavanones, and pterocarpans (Davies and Schwinn, 2006). Their best-known representatives are compounds belonging to the group of isoflavones, which are found in food and agricultural crops such as soy, alfalfa, or red clover. These compounds are used mainly because of their phytoestrogenic effect, and since the wild-grown plants are cultivated in large areas, they are easy to obtain (Clifford and Brown, 2006). In addition to isoflavones, the group of isoflavanones and pterocarpans also include compounds with valuable biological effects; however, the plants that contain them mostly live only in the wild. For example, Ononis species are well-known herbal remedies in the Mediterranean region. As a member of the Fabaceae (Leguminosae) family, they are rich in isoflavonoids, and beside isoflavones, they produce less-prevalent isoflavanones and pterocarpans (Gampe et al., 2016, 2018b). Despite the medicinal benefits (Addotey et al., 2018; Deipenbrock et al., 2020; Spiegler et al., 2020; Stojkovic et al., 2020; Stojković et al., 2020), the plant is not cultivated. The part of the herb mostly used in phytomedicine is the extremely hardy root, making the collection cumbersome. Regarding these aspects, biotechnological methods could play a role in the larger-scale yield of Ononis isoflavonoids. Tumova et al. investigated the flavonoid content of callus cultures and cell suspensions of Ononis arvensis, but their experiments covered only the measurement of total flavonoid content in elicited cultures (Tůmová and Rusková, 1998; Tůmová et al., 2003, 2011; Tůmová and Polívková, 2006). Regarding this information, we aimed to analyze the isoflavonoid profile of hairy root cultures qualitatively and quantitatively, in order to define their capacity to produce biologically valuable isoflavonoids.

\section{MATERIALS AND METHODS}

\section{General Materials}

Standard compound naringenin were purchased from SigmaAldrich (St. Louis, MO, United States), and formononetin, pseudobaptigenin, onogenin, sativanone, medicarpin, and maackiain were purified from hydrolyzed extracts of Ononis spinosa root in our laboratory. The isoflavone glucoside standards (formononetin-, pseudobaptigenin-, onogenin-, sativanone-, maackiain-, and medicarpin glucoside) were isolated in our laboratory, too (Gampe et al., 2020). High-performance liquid chromatography (HPLC) and Mass spectrometry (MS)-grade methanol and acetonitrile were purchased from Fischer Scientific Co. (Fair Lawn, NJ, United States); LiChropur formic- and acetic acid were obtained from Merck (Darmstadt, Germany). Purified water was prepared using a Millipore Direct-Q system (Millipore Corp., Bedford, MA, United States).

\section{Plant Material}

Transformed root cultures of Ononis spinosa L. and Ononis arvensis L. were obtained by the inoculation of sterile 6week-old plants with Agrobacterium rhizogenes (strain R-1601). The segments of hypocotyls with fast-growing adventitious roots were transferred to Petri dishes containing MS medium (Murashige and Skoog, 1962), and cefotaxime was added to the medium $(500 \mathrm{mg} / \mathrm{l})$ for several subcultures until the total disappearance of Agrobacterium (Kuzovkina et al., 1996). After elimination of bacteria, the hairy roots were cultured in liquid Gamborg B5 (Gamborg et al., 1968) media in Erlenmeyer flasks, in a CERTOMAT BS-4 programmable incubation shaking cabinet (Braun Biotech International, Melsungen, Germany) at $100 \mathrm{rpm}$ at $23 \pm 2{ }^{\circ} \mathrm{C}$ in the dark, and were subcultured every 21 days. Genomic DNA was extracted from hairy roots using the protocol and reagents of the Maxwell 16 LEV Plant DNA Kit (Madison, WI, United States). Polymerase chain reaction was executed for the confirmation of the presence of $\mathrm{rolB}$ rooting locus. The primers used to detect rolB were forward 5'-GAAGGTGCAAGCTACCTCTC-3' and reverse $5^{\prime}$ GCTCTTGCAGTGCTAGATTT-3' designed by Furner et al. (1986) (Bio Basic Canada Inc.). A PCR program described by Bertóti et al. (2019) was applied in a Bio-Rad iCycler machine (Hercules, CA, United States). Amplified PCR products were separated using electrophoresis on a $2 \% w / v$ agarose gel (Bio-Rad, CA, United States). From the two species, three parallel samples were inoculated (both 21-day-old, O. spinosa average mass $0.74 \mathrm{~g}$ and $O$. arvensis average mass $0.58 \mathrm{~g}$ ) in $100-\mathrm{ml}$ Erlenmeyer flasks with fresh liquid B5 medium $(40 \mathrm{ml})$ and cultivated in the same shaking cabinet as before mentioned $\left(100 \mathrm{rpm}\right.$ at $23 \pm 2^{\circ} \mathrm{C}$ in the dark). Sampling of plant material was carried out at the time of inoculation $(0 \mathrm{~h})$ and at $7,13,21$, and 28 days after inoculation.

\section{Sample Preparation}

For the qualitative study, $0.10 \mathrm{~g}$ of freeze-dried (Christ Alpha 14 liophilizator, Braun, Melsungen, Germany) and ground hairy root culture was extracted with $5 \mathrm{ml} 70 \%$ methanol using sonication for $30 \mathrm{~min}$ at room temperature. For quantitative analysis, $0.100 \mathrm{~g}$ powdered plant material was weighed and $50 \mu \mathrm{l}$ 
of the internal standard $(2.0 \mathrm{mg} / \mathrm{ml}$ naringenin solution $)$ was added first, and then, the samples were extracted with $5 \mathrm{ml}$ $70 \%$ methanol by sonication for $30 \mathrm{~min}$. The samples were centrifuged, and the pellet was repeatedly extracted twice more with the same method. The collected supernatants were filled up to $25 \mathrm{ml}$, out of which $1 \mathrm{ml}$ was filtered through a $0.22-\mu \mathrm{m}$ PTFE filter (Nantong FilterBio Membrane Co., Ltd., Nantong, Jiangsu, China). From these, $200 \mu \mathrm{l}$ were taken out and kept at $83^{\circ} \mathrm{C}$ for $5 \mathrm{~h}$ prior to HPLC analysis in order to hydrolyze malonate esters (Gampe et al., 2020). Investigating the liquid media for possible exudation of isoflavonoids, it was filtered and analyzed directly.

\section{UHPLC-ESI-Orbitrap-MS/MS Conditions for the Qualitative Analysis of Hairy Root Samples}

For obtaining high-resolution mass spectrometric data of hairy root cultures, a Dionex UltiMate 3000 UHPLC system (3000RS diode array detector, TCC-3000RS column thermostat, HPG-3400RS pump, SRD-3400 solvent rack degasser, and WPS-3000TRS autosampler) was used hyphenated with a Orbitrap Q Exactive Focus Mass Spectrometer equipped with electrospray ionization (Thermo Fisher Scientific, Waltham, MA, United States). The UHPLC separation of the samples was attained on a Waters XSelect CSH Phenyl-Hexyl phase column $(100 \times 2.1 \mathrm{~mm}$ i.d.; $3.5 \mu \mathrm{m}$; Waters Corporation, Milford, MA, United States). Mobile phase consisted of $0.1 \% v / v$ formic acid (A) and 8:2 acetonitrile:0.1\% $v / v$ formic acid (B). The following gradient program was applied: $0 \mathrm{~min}, 20 \% \mathrm{~B} ; 15 \mathrm{~min}$, $80 \% \mathrm{~B} ; 20 \mathrm{~min}, 80 \% \mathrm{~B}$; and $22 \mathrm{~min}, 20 \% \mathrm{~B}$. Solvent flow rate was $0.3 \mathrm{ml} / \mathrm{min}$, and the column temperature was set to $25^{\circ} \mathrm{C}$. The injection volume was $2 \mu$ l. The electrospray ionization source was operated in positive ionization mode, and operation parameters were optimized automatically using the built-in software. The working parameters were as follows: spray voltage, $3,500 \mathrm{~V}$; capillary temperature, $256.25^{\circ} \mathrm{C}$; sheath gas $\left(\mathrm{N}_{2}\right), 47.5^{\circ} \mathrm{C}$; auxiliary gas $\left(\mathrm{N}_{2}\right), 11.25$ arbitrary units; and spare gas $\left(\mathrm{N}_{2}\right), 2.25$ arbitrary units. The resolution of the full scan was of 70,000, and the scanning range was between 120 and $1,000 \mathrm{~m} / z$ units. The most intense ions detected in full scan spectrum were selected for MS/MS scan at a resolving power of 35,000, in the range of 50$1,000 \mathrm{~m} / \mathrm{z}$ units. Parent ions were fragmented with normalized collision energy of $10 \%, 30 \%$, and $45 \%$.

\section{Isolation of Bulatlactone 2 "-O- $\beta$-D-Glucoside and Ononilactone}

Using an ultrasonic bath, $4.5 \mathrm{~g}$ of lyophilized, powdered sample of 4-week-old hairy root cultures of $O$. spinosa were extracted with $200 \mathrm{ml} \mathrm{50 \%} \mathrm{methanol} \mathrm{for} 30 \mathrm{~min}$. The extract was filtered and dried under reduced pressure at $60^{\circ} \mathrm{C}$. The residue was redissolved in $5 \mathrm{ml} \mathrm{30 \%} \mathrm{methanol} \mathrm{and} \mathrm{purified} \mathrm{using} \mathrm{the} \mathrm{same}$ flash chromatographic method mentioned at the isolation of standard compounds. The fractions eluting between 2 and $3 \mathrm{~min}$ were unified and further separated on a preparative HPLC system using eluents of $0.3 \% v / v$ acetic acid (A) and methanol (B). Gradient elution was used with the following program: $0 \mathrm{~min}$, $30 \% \mathrm{~B} ; 10 \mathrm{~min}, 30 \% \mathrm{~B}$; and $20 \mathrm{~min}, 100 \% \mathrm{~B}$ with a $10-\mathrm{ml} / \mathrm{min}$ flow rate. Bulatlactone eluted at $7.5 \mathrm{~min}$ and the yield was $12.1 \mathrm{mg}$. From flash chromatography fractions eluted between 13 and $14 \mathrm{~min}$, ononilactone was isolated using the same preparative HPLC system with the following eluents: $0.3 \% v / v$ acetic acid (A) and acetonitrile (B). The used gradient was as follows: 0 min $40 \%$ $\mathrm{B}$ up to $43 \%$ in $20 \mathrm{~min}$. The peak eluted at $11.8 \mathrm{~min}$ was further purified using an isocratic method consisting of $63 \%$ methanol and $37 \% 0.3 \% v / v$ acetic acid with a $10-\mathrm{ml} / \mathrm{min}$ flow rate. The peak of interest eluted at $12.5 \mathrm{~min}$ and the yield was $1.4 \mathrm{mg}$.

\section{Nuclear Magnetic Resonance Spectroscopy}

Nuclear magnetic resonance (NMR) spectral studies for ononilactone were carried out on Avance III HDX spectrometer from Bruker BioSpin GmbH (Rheinstetten, Germany): $800 \mathrm{MHz}$ (equipped with a ${ }^{1} \mathrm{H} \&{ }^{19} \mathrm{~F} /{ }^{13} \mathrm{C} /{ }^{15} \mathrm{~N}$ TCI CryoProbe ${ }^{1} \mathrm{H}$ : $799.7 \mathrm{MHz},{ }^{13} \mathrm{C}$ : $201.0 \mathrm{MHz}$ ) and $500 \mathrm{MHz}$ (with a $500 \mathrm{~S} 2$ ${ }^{1} \mathrm{H} /{ }^{13} \mathrm{C} /{ }^{15} \mathrm{~N}$ TCI Extended Temperature CryoProbe, ${ }^{1} \mathrm{H}$ : 499.9 MHz, ${ }^{13} \mathrm{C}$ : $125.7 \mathrm{MHz}$ ). Standard pulse sequences available in the TopSpin $3.5 \mathrm{pl} 7$ software were used for spectral acquisition, while the spectra were processed in MestreNova (Mestrelab Research). The complete resonance assignments were established from scalar and through-space ${ }^{1} \mathrm{H}-{ }^{1} \mathrm{H}$, direct ${ }^{1} \mathrm{H}-{ }^{13} \mathrm{C}$, and long-range ${ }^{1} \mathrm{H}-{ }^{13} \mathrm{C}$ connectivities on the basis of $1 \mathrm{D}{ }^{1} \mathrm{H}$, ${ }^{13} \mathrm{C}$ as well as $2 \mathrm{D}$ COSY, ROESY (CW spinlock for $250 \mathrm{~ms}$ ), ${ }^{1} \mathrm{H}-{ }^{13} \mathrm{C}$ multiplicity-edited HSQC $\left({ }^{1} J_{\mathrm{CH}}=140 \mathrm{~Hz}\right)$, and ${ }^{1} \mathrm{H}_{-}{ }^{13} \mathrm{C}$ HMBC $\left({ }^{n} J_{\mathrm{CH}}=7\right.$ and $\left.2.5 \mathrm{~Hz}\right)$ spectra, respectively. The sample temperature was maintained at $298 \mathrm{~K}$, and standard 5-mm NMR tubes were used. The ${ }^{1} \mathrm{H}$ and ${ }^{13} \mathrm{C}$ chemical shifts were referenced to the solvent signal of $\mathrm{CHD}_{2} \mathrm{SOCD}_{3}$ at $\delta_{H}=2.500 \mathrm{ppm}$ and the resonance line of $\mathrm{CD}_{3} \mathrm{SOCD}_{3}$ at $\delta_{C}=39.520 \mathrm{ppm}$, respectively. The sample was dissolved in $600 \mu \mathrm{l}$ DMSO- $d_{6}$ (VWR International L.L.C.) and acidified by two drops of neat trifluoroacetic acid (TFA). NMR experiments for the structural analysis of bulatlactone $2^{\prime \prime}-O-\beta$-D-glucoside were carried out in $\mathrm{D}_{2} \mathrm{O}$ on a $600-\mathrm{MHz}$ Varian DDR NMR spectrometer (Agilent Technologies, Palo Alto, CA, United States) equipped with a 5 -mm inverse-detection gradient (IDPFG) probehead. Standard pulse sequences and processing routines available in VnmrJ $3.2 \mathrm{C} /$ Chempack 5.1 were used for the structure identification. The complete resonance assignments were established from scalar and through-space ${ }^{1} \mathrm{H}_{-}{ }^{1} \mathrm{H}$, direct ${ }^{1} \mathrm{H}_{-}{ }^{13} \mathrm{C}$, and long-range ${ }^{1} \mathrm{H}-{ }^{13} \mathrm{C}$ connectivities as described above. The probe temperature was maintained at $298 \mathrm{~K}$, and standard 5-mm NMR tubes were used. The ${ }^{1} \mathrm{H}$ chemical shifts were referenced to the residual solvent signal $\delta_{H}=4.790 \mathrm{ppm}$.

\section{Preparation of Stock Solutions, Calibration Standards, and Quality Control Samples}

Individual stock solutions of the standards were prepared by dissolving the compounds in $70 \%$ methanol containing the internal standard $(50 \mu 12.0 \mathrm{mg} / \mathrm{ml}$ naringenin solution diluted to $25 \mathrm{ml}$ ) to obtain $\sim 1 \mathrm{mg} / \mathrm{ml}$ solutions. Equal parts of the standard solutions were mixed to gain the stock solution. Calibration standards were prepared by diluting the stock solution with 
the solution of the internal standard. The 10-point calibration curve was prepared using the following: 100, 60, 30, 10, 6, 3, $1,0.6,0.3$, and $0.1 \mu \mathrm{g} / \mathrm{ml}$ concentration levels. QC samples were prepared separately from the stock solution at 50,5, and $0.5 \mu \mathrm{g} / \mathrm{ml}$ nominal concentrations.

\section{UHPLC-UV-DAD Conditions for the Quantitative Analysis of Ononis Samples}

Quantitative measurements were executed on a Waters ACQUITY UPLC system (sample manager, binary solvent manager, and PDA detector) (Waters Corporation, Milford, MA, United States). The samples were analyzed using the same phenyl-hexyl column as mentioned at the qualitative studies. Aiming at the determination of isoflavone derivatives, the same eluents were used, with the following gradient program: $0 \mathrm{~min}$, $10 \% \mathrm{~B} ; 15 \mathrm{~min}, 30 \% \mathrm{~B} ; 17 \mathrm{~min}, 100 \% \mathrm{~B}$; and $19 \mathrm{~min}, 10 \% \mathrm{~B}$ with $0.4 \mathrm{ml} / \mathrm{min}$ flow rate and $5 \mu \mathrm{l}$ injected volume, and the column was heated to $40^{\circ} \mathrm{C}$. For quantification of the isoflavanone and pterocarpan derivatives, the following gradient was used: $0 \mathrm{~min}$, $25 \%$ B; $5 \mathrm{~min}, 25 \%$; $6 \mathrm{~min}, 29 \%$ B; $15 \mathrm{~min}, 29 \%$; $17 \mathrm{~min}, 100 \% \mathrm{~B}$; and $19 \mathrm{~min}, 25 \% \mathrm{~B}$ with $0.4 \mathrm{ml} / \mathrm{min}$ flow rate and $5 \mu \mathrm{l}$ injected volume, and the column was heated to $27^{\circ} \mathrm{C}$ (Gampe et al., 2020).

\section{RESULTS}

\section{Characterization of Hairy Root Growth}

For both Ononis species, the complete and stable transformation status of the isolated hairy root cultures (HRCs) was confirmed by PCR on their genomic DNA for the detection of the presence of the proto-oncogene rolB. In general, the HRCs of $O$. spinosa showed a more robust phenotype and darker color, than O. arvensis (Figure 1). Investigating the change in biomass, a very similar trend could be observed. The biomass of both species increased until the $3 \mathrm{rd}$ week. In the first 2 weeks, the increase was lighter in the case of $O$. arvensis. During the 3 rd week, both cultures reached their maximum in biomass production and their growth did not differ significantly. Reaching the 4th week, the biomass started to decrease, showing the aging of the cultures. The dry masses showed a similar pattern; however, O. spinosa cultures showed a significantly higher dry mass. O. arvensis HRCs followed a similar trend, but the change in the dry mass is not significant (Figure 2).

\section{Qualitative Characterization of Phytochemical Composition of Ononis HRCs}

The total ion chromatogram (TIC) recorded in positive ionization mode of the $70 \%$ aqueous-methanol extracts with the identified compounds of O. spinosa and O. arvensis HRCs can be seen in Figure 3.

The identified compounds along with their number, retention time, protonated pseudo-molecular ions, and most intense product ions are shown in Supplementary Table 1. All identified compounds could be observed in both species.
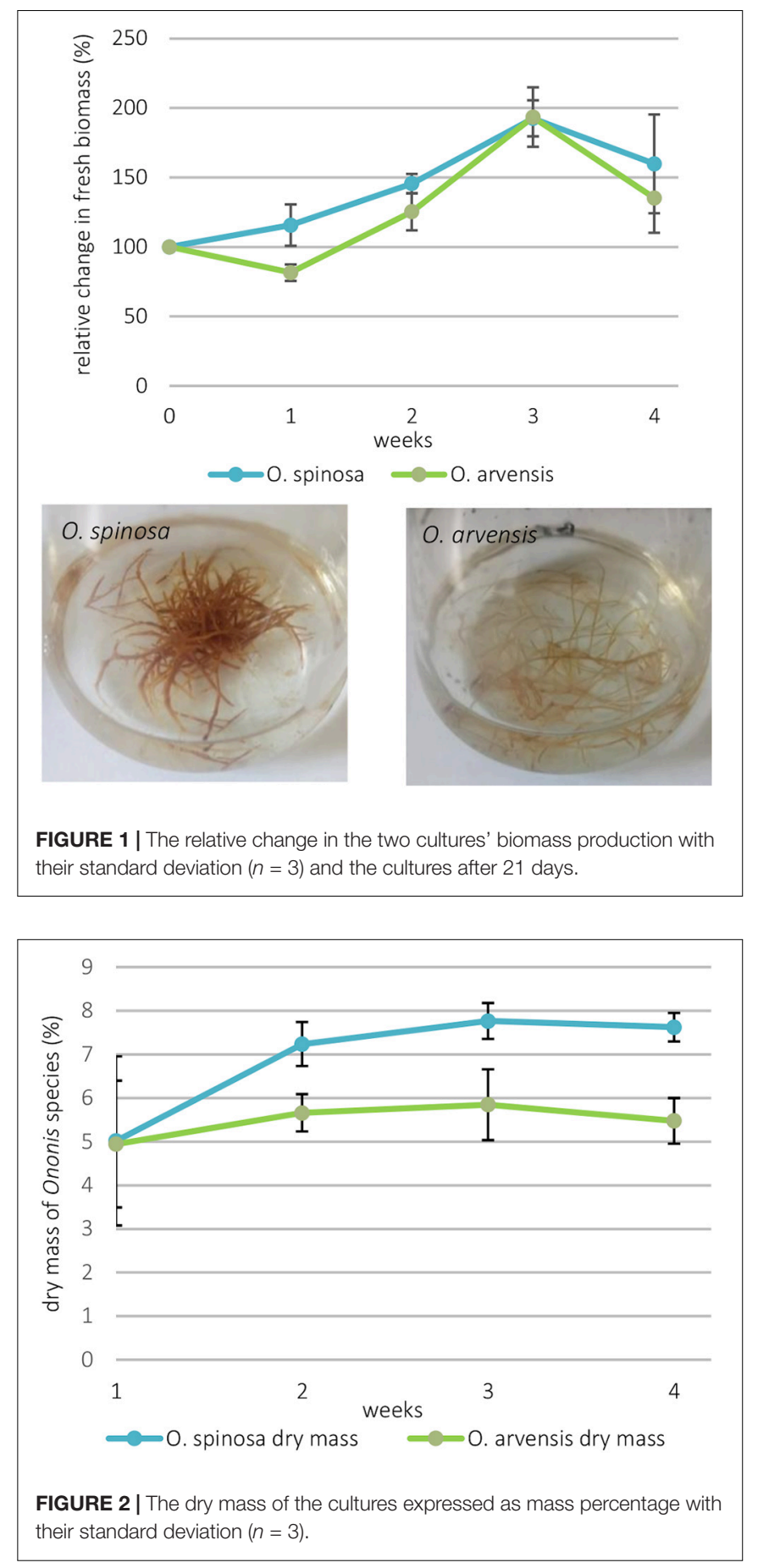

The most characteristic peaks of the samples were isoflavonoid derivatives. Isoflavonoid derivatives were identified following the methods described in our previous publication (Gampe et al., 2016, 2018a,b). In the HRCs, the isoflavonoid derivatives could be found in the forms of glucosides $(10,12,13,14,16$, and 17), glucoside malonates $(17,20,21$, and 22$)$, aglycones $(24,25,27$, 28, and 29), and homopipecolic acid esters of glucosides (5, 6, 7, and 8) (Supplementary Table 1). Based on solely HR-MS/MS studies, the type of hexoside and the position of the malonate 


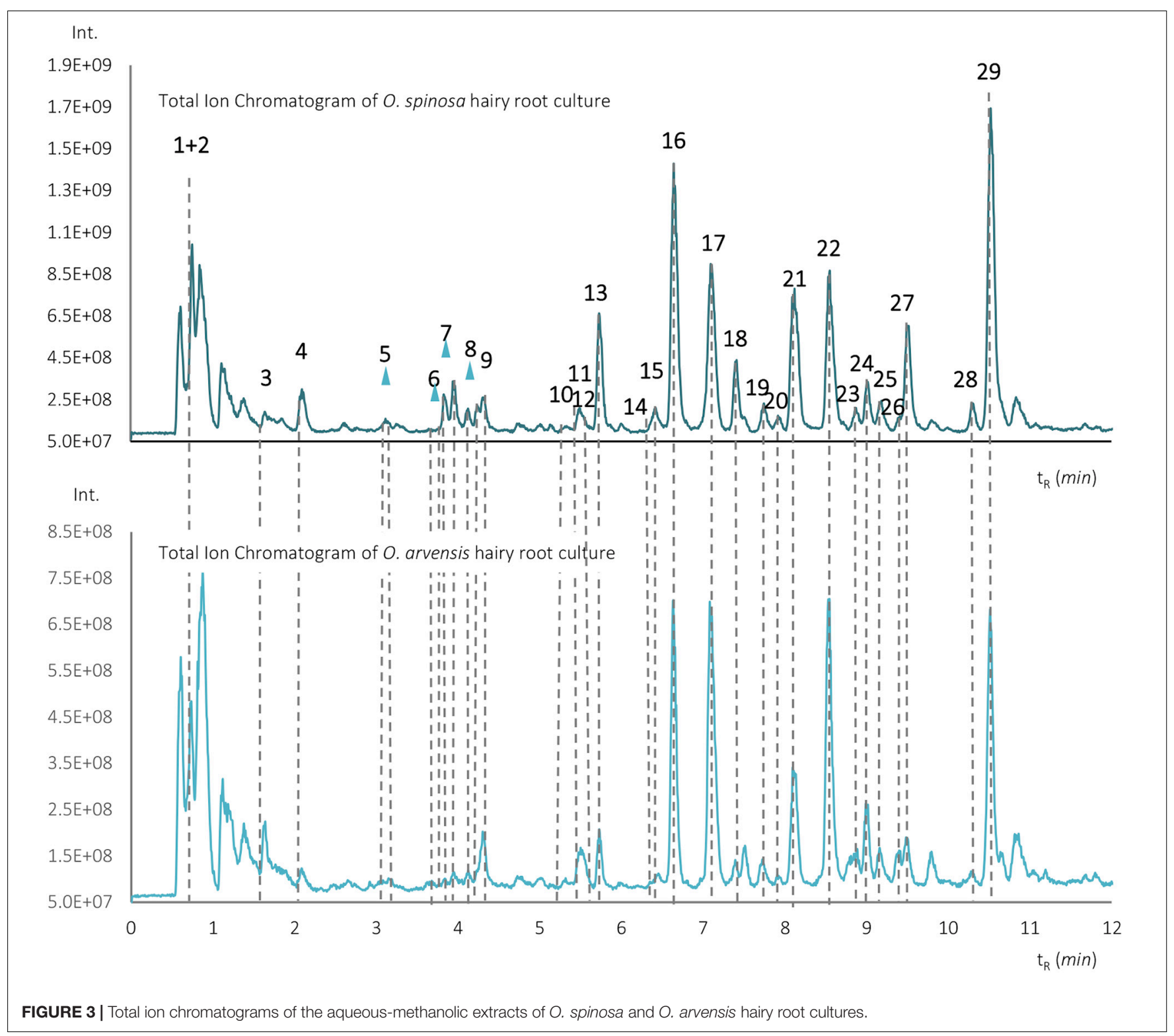

moiety could not be deduced, so the compounds were identified tentatively as 7-O-glucosides and 7-O-glucoside $6 "$-O-glucoside malonates based on the works of Farag et al. (2007) and de Rijke et al. (2004). Because of the racemic feature of the beta amino acid moiety, the homopipecolic acid derivatives can be found in the form of diastereomeric pairs separated to double peaks on the stationary phase (Supplementary Table 1). The first two peaks were identified as the methyl-esters of homoproline and homopipecolic acid; the esterification of homoproline in methanol as extraction solvent has already been reported before (Paßreiter, 1992). Although homoproline could be found in the form of methyl ester, the corresponding homoproline isoflavonoid glucoside esters could not be detected.

Beside isoflavonoid derivatives, special phenolic lactones (norneolignans) were detected in the forms of glucosides (9 and 11) and aglycones (15 and 23). These derivatives of puerol
A and clitorienolactone B were known in Ononis species before (Ghribi et al., 2015; Addotey et al., 2018; Gampe et al., 2018b). Surprisingly, a compound (peak 26) with a different fragmentation profile and a rather unusual UV spectrum with two absorption maxima was also observed eluting with the aglycones (Figure 4).

The solution of this compound in aqueous-polar organic solvents and DMSO showed a strong blue fluorescence if irradiated with UV light of $366 \mathrm{~nm}$. The most dominant fragment ions originated from the sequential losses of $\mathrm{CO}$ and $\mathrm{H}_{2} \mathrm{O}$ units. Based on HR-MS analysis, the observed protonated pseudomolecular ion showed an $\mathrm{m} / \mathrm{z} 295.0591$ value, and the calculated formula was $\mathrm{C}_{17} \mathrm{H}_{10} \mathrm{O}_{5}$, suggesting the presence of 13 double bond equivalents. Since broadened aromatic ${ }^{1} \mathrm{H}$ NMR signals could be observed in DMSO- $d_{6}$, hindering the observation of key ${ }^{1} \mathrm{H}_{-}{ }^{13} \mathrm{C}$ correlations in the heteronuclear $2 \mathrm{D}$ spectra, two drops 


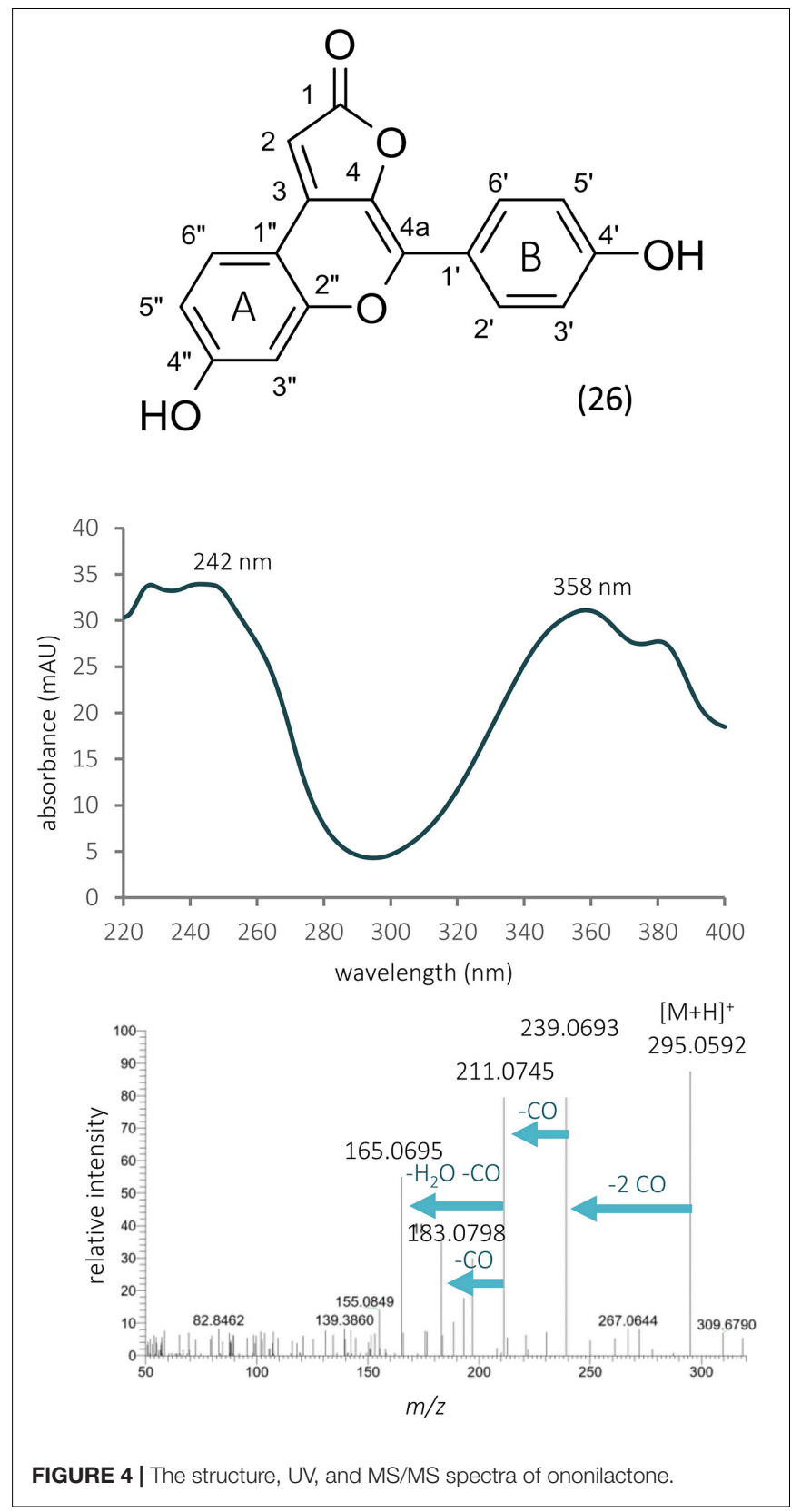

of TFA was applied to overcome line broadening. NMR spectra of the acidified spectrum enabled the structure elucidation of compound 26 from only $49 \mu \mathrm{M}$ material (ca. $9 \mu \mathrm{g}$ in the NMR tube). In the ${ }^{13} \mathrm{C} \mathrm{NMR}$ spectrum, the presence of a carbonyl group at $\delta_{C} 170.5$; six quaternary carbons at $\delta_{C} 163.2,160.0,146.4$, $135.4,134.1,120.0$, and 107.4 ; one methine carbon at $\delta_{C} 89.1$; and nine aromatic methine carbons between $\delta_{C} 103.0$ and 129.8 were confirmed (Table 1). The ${ }^{1} \mathrm{H}$ NMR contained spectrum seven aromatic and one methine proton (see Table $\mathbf{1}$ ).

Investigating the early eluting peaks, peak 4 showed a UV spectrum very similar to that of chlorogenic acid or other caffeoyl acid derivatives (Figure 5). Moreover, in the HR-MS spectrum, a peak at $m / z 355.1054$ could be detected, which could result in
TABLE 1 $\mid{ }^{1} \mathrm{H},{ }^{13} \mathrm{C}$, and 2D NMR data of ononilactone in DMSO- $\mathrm{d}_{6}$ acidified by TFA [ $\delta(\mathrm{ppm}), J(\mathrm{~Hz})]$.

\begin{tabular}{lccc}
\hline No. & $\mathbf{1}^{\mathbf{H}}$ & ${ }^{\mathbf{1 3}} \mathbf{C}$ & HMBC \\
\hline $\mathbf{1}$ & - & 170.5 & \\
$\mathbf{2}$ & $6.07 \mathrm{~s}$ & 89.1 & $170.5,146.4,107.4$ \\
$\mathbf{3}$ & - & 146.4 & \\
$\mathbf{4}$ & - & 134.1 & \\
$\mathbf{4 a}$ & - & 135.4 & \\
$\mathbf{1}^{\prime}$ & - & 120.0 & \\
$\mathbf{2}^{\prime}$ & $8.01 \mathrm{~d} J=8.4$ & 129.0 & $129.0,135.4,160.0$ \\
$\mathbf{3}^{\prime}$ & $7.00 \mathrm{~d} J=8.4$ & 116.2 & $120.0,116.2,160.0$ \\
$\mathbf{4}^{\prime}$ & - & 160.0 & \\
$\mathbf{5}^{\prime}$ & $7.00 \mathrm{~d} J=8.4$ & 116.2 & $120.0,116.2,160.0$ \\
$\mathbf{6}^{\prime}$ & $8.01 \mathrm{~d} J=8.4$ & 129.0 & $129.0,135.4,160.0$ \\
$\mathbf{1}^{\prime \prime}$ & - & 107.4 & \\
$\mathbf{2}^{\prime \prime}$ & - & 152.7 & \\
$\mathbf{3}^{\prime \prime}$ & $7.02 \mathrm{~d} J=2.3$ & 103.0 & $107.4,115.4,153.0,161.8$ \\
$\mathbf{4}^{\prime \prime}$ & - & 161.8 & \\
$\mathbf{5}^{\prime \prime}$ & $6.98 \mathrm{dd} J=8.5,2.3$ & 115.4 & $107.4,103.0$ \\
$\mathbf{6}^{\prime \prime}$ & $7.94 \mathrm{~d} J=8.5$ & 128.0 & $146.4,152.7,163.2$ \\
\hline
\end{tabular}

the same molecular formula $\left(\mathrm{C}_{16} \mathrm{H}_{18} \mathrm{O}_{9}\right)$ as chlorogenic acid, but the fragmentation profile did not match. The ${ }^{1} \mathrm{H}$ NMR spectrum revealed glucose resonances $\left(\delta_{H} 3.54,3.62,3.70,3.70,3.80,3.98\right.$, and 5.20) and two aromatic spin systems. Similarly to compound 26 , a para-disubstituted phenyl ring gives the resonances at $\delta_{H}$ 6.71 and $6.87 \mathrm{ppm}$, while the multiplets at $\delta_{H} 6.67,6.79$, and 7.25 indicate a 1,2,4 trisubstituted phenyl ring (Table 2). Three singlet signals were also recorded at $\delta_{H} 5.19,6.07$, and 6.22. The lack of scalar coupling between the adjacent methine protons may be the consequence of their ca. 90 dihedral angle, according to the Karplus relationship.

\section{Quantitative Analysis of Isoflavonoids in Ononis HRCs}

Using the same UHPLC-UV-DAD method developed for the characterization of wild-grown Ononis species, the relative and absolute isoflavonoid contents of HRCs were evaluated (Supplementary Tables 2, 3). Firstly, the liquid media were investigated, but no isoflavonoid derivatives could be detected. In the in vitro cultures, the main compounds were sativanone glucoside and medicarpin glucoside followed by pseudobaptigenin glucoside and formononetin glucoside (Figure 6). The aglycones could be observed in a magnitude lower quantity for sativanone and medicarpin, whereas in the case of isoflavones, they were under limit of detection (Supplementary Tables 2, 3). In O. spinosa samples, the isoflavonoid concentration $(\mathrm{mg} / 100 \mathrm{mg})$ showed a constant regression from the 1st week, whereas in O. arvensis samples, up to the 2nd week the level increased, then dropped (Figure 7). The O. spinosa cultures possessed a higher level of total isoflavonoid content than $O$. arvensis (Figures 7,8 ). If the absolute quantities of isoflavonoids were investigated (Figure 8), without the correction of the biomass, both samples reached their maximum at $3 \mathrm{rd}$ week (similarly to the biomass). In the 4 th week, the 


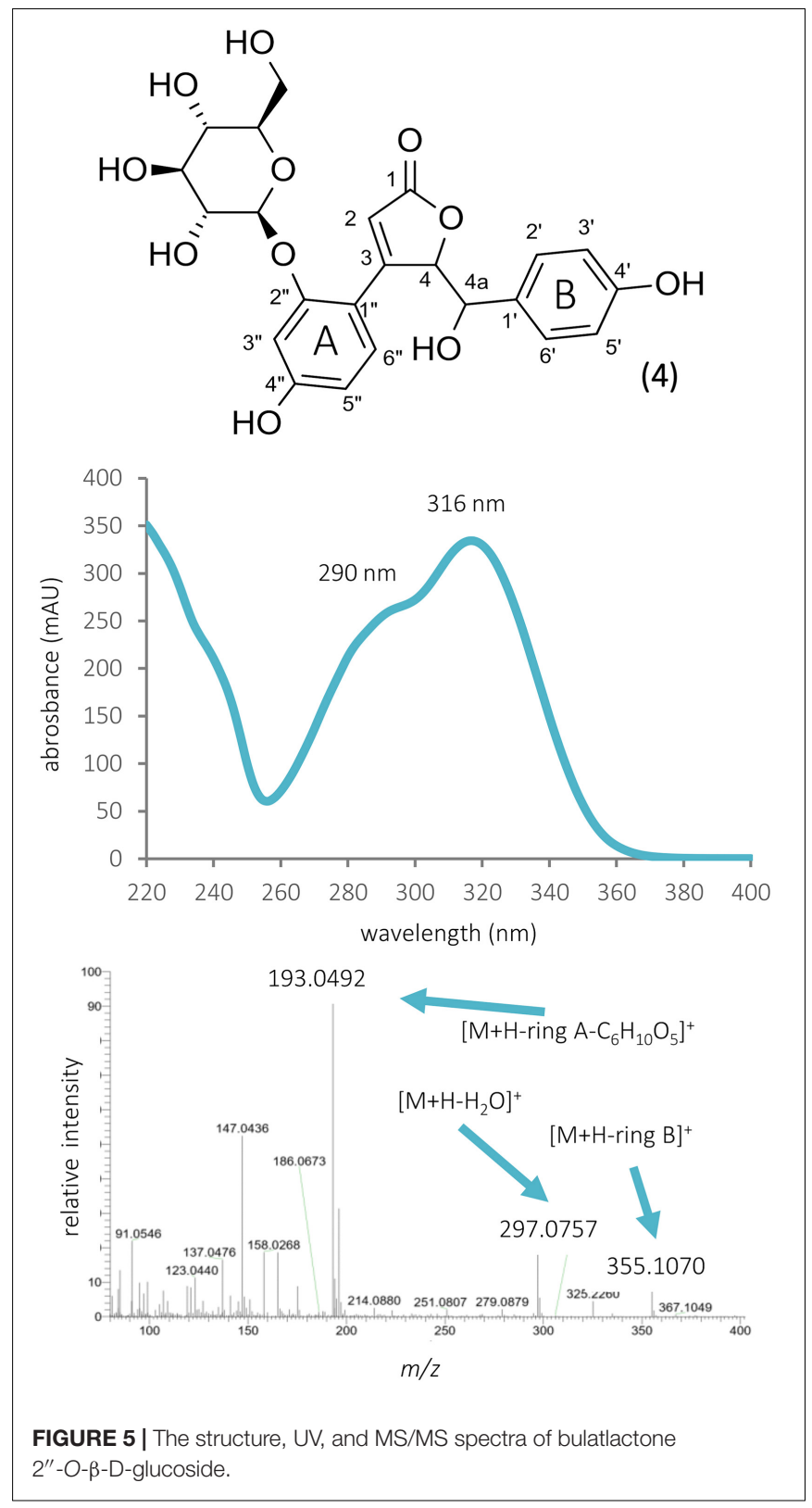

isoflavonoid levels dropped, indicating their breakdown or transformation (Figure 8).

\section{DISCUSSION}

\section{Structural Identification of the New Compounds}

The UV spectrum of compound 26 resembled to that of flavonoids; however, the bands did not completely overlap with that of standard compounds (Markham and Mabry, 1975). The MS/MS fragmentation spectrum recorded in positive ionization mode did not show any specific patterns characteristic for flavonoids or isoflavonoids (Cuyckens and Claeys, 2004). The<smiles></smiles>

FIGURE 6 | The most abundant structures found in Ononis HRC extracts.

TABLE $2 \mid{ }^{1} \mathrm{H},{ }^{13} \mathrm{C}$, and 2D NMR data of bulatlactone $2^{\prime \prime}-\mathrm{O}-\beta$-D-glucoside in $\mathrm{D}_{2} \mathrm{O}$ [ $\delta(\mathrm{ppm}), J(\mathrm{~Hz})]$.

\begin{tabular}{|c|c|c|c|}
\hline No. & ${ }^{1} \mathrm{H}$ & ${ }^{13} \mathrm{C}$ & HMBC \\
\hline 1 & - & 177.0 & \\
\hline 2 & $6.07 \mathrm{~s}$ & 113.9 & $87.1,111.8,164.1,176.9$ \\
\hline 3 & - & 164.1 & \\
\hline 4 & $6.22 \mathrm{~s}$ & 87.1 & $73.4,113.9,127.8,164.1,176.9$ \\
\hline $4 a$ & $5.19 \mathrm{~s}$ & 73.4 & $127.8,128.6$ \\
\hline $1^{\prime}$ & - & 127.8 & \\
\hline $2^{\prime}$ & $6.87 \mathrm{~d} J=8.4$ & 128.6 & $73.4,114.5,128.6,155.4$ \\
\hline $3^{\prime}$ & $6.71 \mathrm{~d} J=8.4$ & 114.5 & $127.8,114.5,155.4$ \\
\hline $4^{\prime}$ & - & 155.4 & \\
\hline $5^{\prime}$ & $6.71 \mathrm{~d} J=8.4$ & 114.5 & $127.8,114.5,155.4$ \\
\hline $6^{\prime}$ & $6.87 \mathrm{~d} J=8.4$ & 128.6 & $73.4,114.5,128.6,155.4$ \\
\hline $1^{\prime \prime}$ & - & 111.8 & \\
\hline $2^{\prime \prime}$ & - & 156.1 & \\
\hline $3^{\prime \prime}$ & $6.79 \mathrm{~d} J=1.7$ & 102.5 & $111.8,156.1,160.5$ \\
\hline $4^{\prime \prime}$ & - & 160.5 & \\
\hline $5^{\prime \prime}$ & $6.67 \mathrm{dd} J=8.5,1.7$ & 110.5 & $102.5,111.8$ \\
\hline $6^{\prime \prime}$ & $7.25 \mathrm{~d} J=8.5$ & 131.8 & $156.1,160.5,164.1$ \\
\hline G1 & $5.20 \mathrm{~d} J=9.4$ & 99.6 & 156.1 \\
\hline G2 & $3.70 \mathrm{t} J=9.4$ & 72.7 & \\
\hline G3 & $3.62 \mathrm{t} \mathrm{J}=9.4$ & 75.8 & \\
\hline G4 & $3.54 \mathrm{t} J=9.4$ & 69.2 & \\
\hline G5 & $3.70 \mathrm{t} J=9.4$ & 76.2 & \\
\hline G6a & $3.80 \mathrm{~d} J=12.0$ & 60.5 & \\
\hline G6b & $3.98 \mathrm{dd} J=12.0,5.7$ & & \\
\hline
\end{tabular}

presence of two phenyl rings with para (ring B) and orthopara (ring A) substitution patterns could be deduced from 

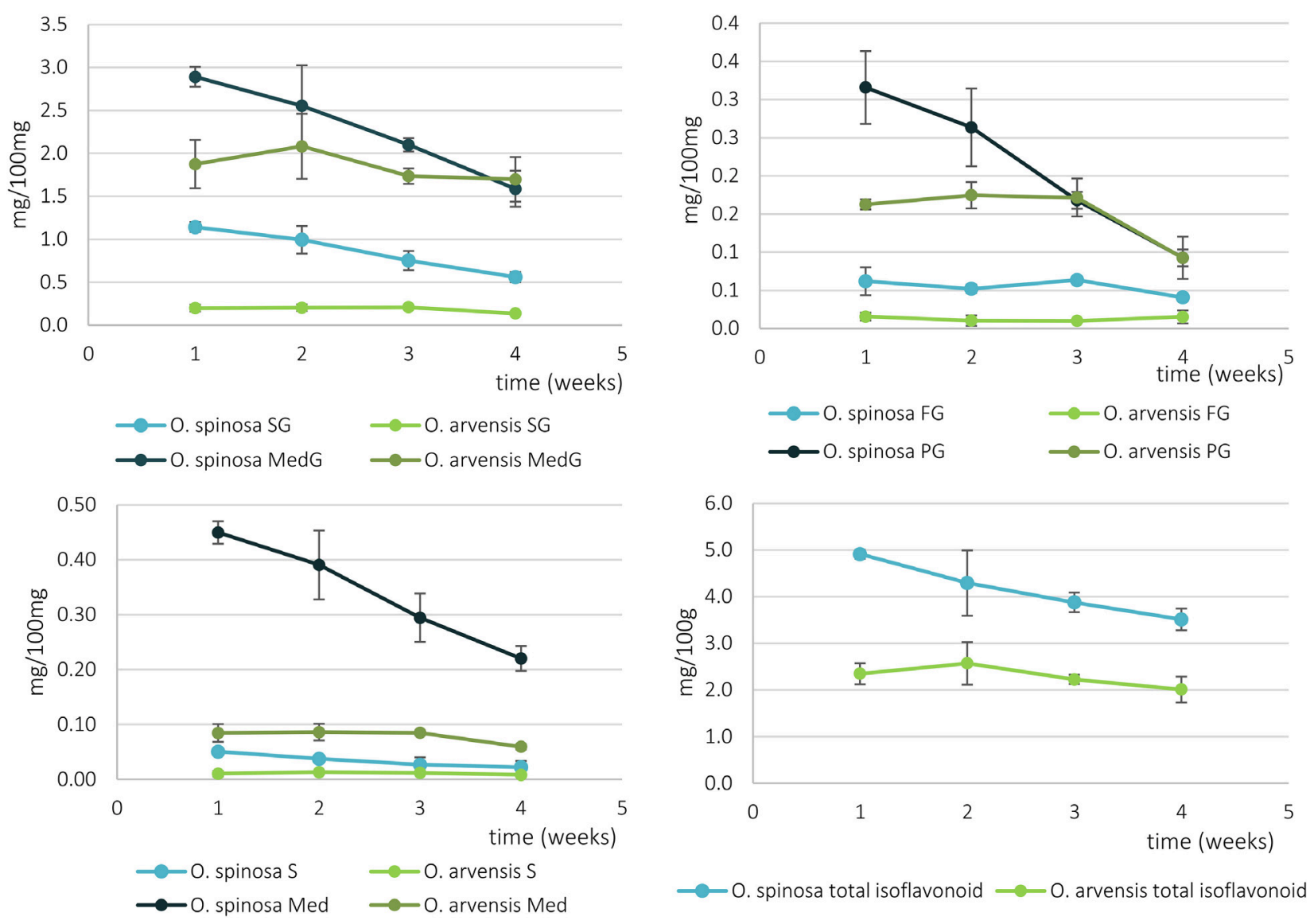

FIGURE 7 | The relative isoflavonoid content of $O$. spinosa and O. arvensis HRCs in mg/100 mg (SG, sativanone glucoside; MedG, medicarpin glucoside; S, sativanone; Med, medicarpin; PG, pseudobaptigenin glucoside; FG, formononetin glucoside).

the coupling constants of ${ }^{1} \mathrm{H}$ resonances and ${ }^{1} \mathrm{H}^{1}{ }^{1} \mathrm{H}$ COSY experiments. Based on the ${ }^{1} \mathrm{H}$ NMR chemical shifts and $2 \mathrm{D}$ HMBC correlation peaks, the protons at $\delta_{H} 8.01$ were shown to belong to $\mathrm{H}-2^{\prime}$ and $6^{\prime}$, while protons at $\delta_{H} 7.00$ to $\mathrm{H}-3^{\prime}$ and $5^{\prime}$ of ring $\mathrm{B}$, respectively. The coupling pattern of the ${ }^{1} \mathrm{H}$ resonances at $\delta_{H} 7.94,7.02$, and 6.98 assigns these chemical shifts to $\mathrm{H}-3^{\prime \prime}$, $\mathrm{H}-6^{\prime \prime}$, and $\mathrm{H}-5^{\prime \prime}$ (ring A), respectively. These aromatic protons served as good entry points to assign the quaternary carbon atoms of ring B. Using the HMBC data, the C-1", C-2", and C$4^{\prime \prime}$ atoms were assigned to peaks at $\delta_{C} 107.4, \delta_{C} 152.7$, and $\delta_{C}$ $163.2 \mathrm{ppm}$, respectively. The sharp singlet at $\delta_{H} 6.07$ showed a HMBC correlation peak to C- $1^{\prime \prime}$, indicating a connection of a $\mathrm{C}=\mathrm{CH}-$ unit to ring $\mathrm{A}$. The position of the $\mathrm{H}-2$ proton has also been confirmed by the ROESY crosspeak of $\mathrm{H}-6^{\prime \prime}$ and $\mathrm{H}$ 2. The same proton $\left(\delta_{H} 6.07\right)$ showed a HMBC crosspeak to the carbonyl $\delta_{C} 170.5$, which permitted the deduction that this olefinic group is linked to an ester or lactone. The structural motif, which could be drawn up regarding the information gained from the NMR experiments, showed a close similarity with the structure of puerol A (Ghribi et al., 2015), so that the presence of a $\delta$-lactone was presumed, which could be confirmed by the HMBC correlations of $\mathrm{H}-2$ with both $\mathrm{C}-3$ and C-4. In the case of puerol derivatives or clitorienolactones (Addotey et al., 2018; Gampe et al., 2018b), one of the phenolic rings (ring A) is linked to the lactone ring through a methylene group. However, in our case, a $\mathrm{CH}_{2}$ unit could not be observed, and the other phenolic ring (ring B) showed a linkage through a quaternary carbon atom based on HMBC experiments. Regarding the molecular formula and the structures of puerol derivatives, an ether bridge between C- 2 " and $\mathrm{C}-4 \mathrm{a}$ was hypothesized. With this linkage, a furanoflavonoid-like structure is formed (Figure 4), which can explain the similarity of the UV spectrum and the blue fluorescence. As this compound is described for the first time, the name ononilactone was chosen for this new skeleton.

Although the UV spectrum, HR-MS base peak, and the calculated formula strongly resembled to chlorogenic acid, looking at the MS/MS spectrum of compound 4, the most intense fragment rose at $m / z$ 193.0491, which was in disagreement with the MS/MS data of chlorogenic acid registered by other research groups [Metabocard of Chlorogenic Acid (HMDB0003164), 2006]. The mass difference $(162 \mathrm{Da})$ between the two peaks (Figure 5) led us to the assumption that this compound is a glycoside, which could lose a hexose unit as a neutral loss. With the same exact mass and molecular formula, scopolin 

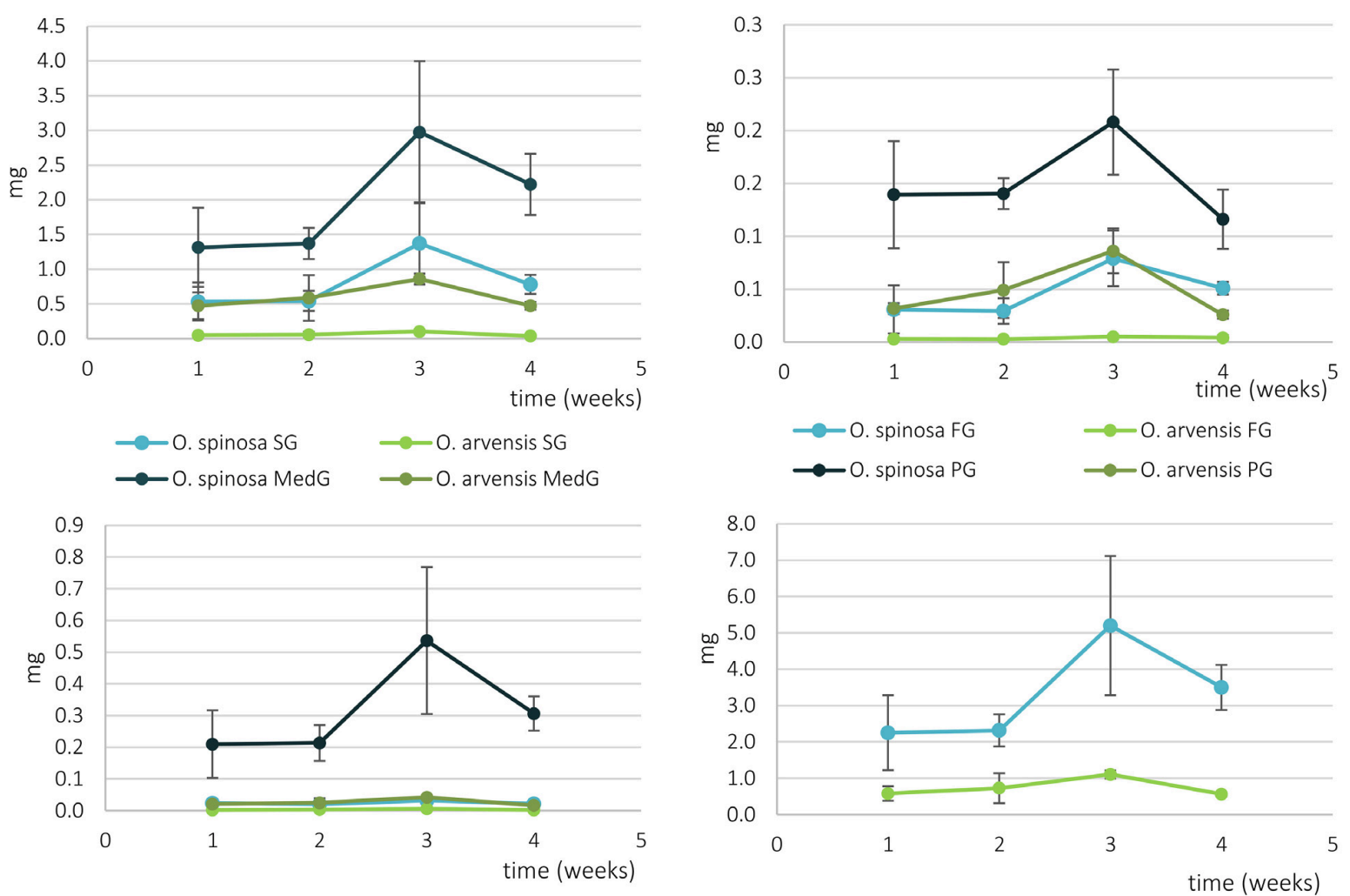

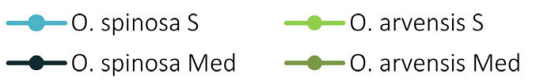

$\longrightarrow$ O. spinosa total isoflavonoid $\longrightarrow 0$. arvensis total isoflavonoid

FIGURE 8 | The absolute isoflavonoid content of O. spinosa and O. arvensis HRCs in milligrams (SG, sativanone glucoside; MedG, medicarpin glucoside; S, sativanone; Med, medicarpin; PG, pseudobaptigenin glucoside; FG, formononetin glucoside).

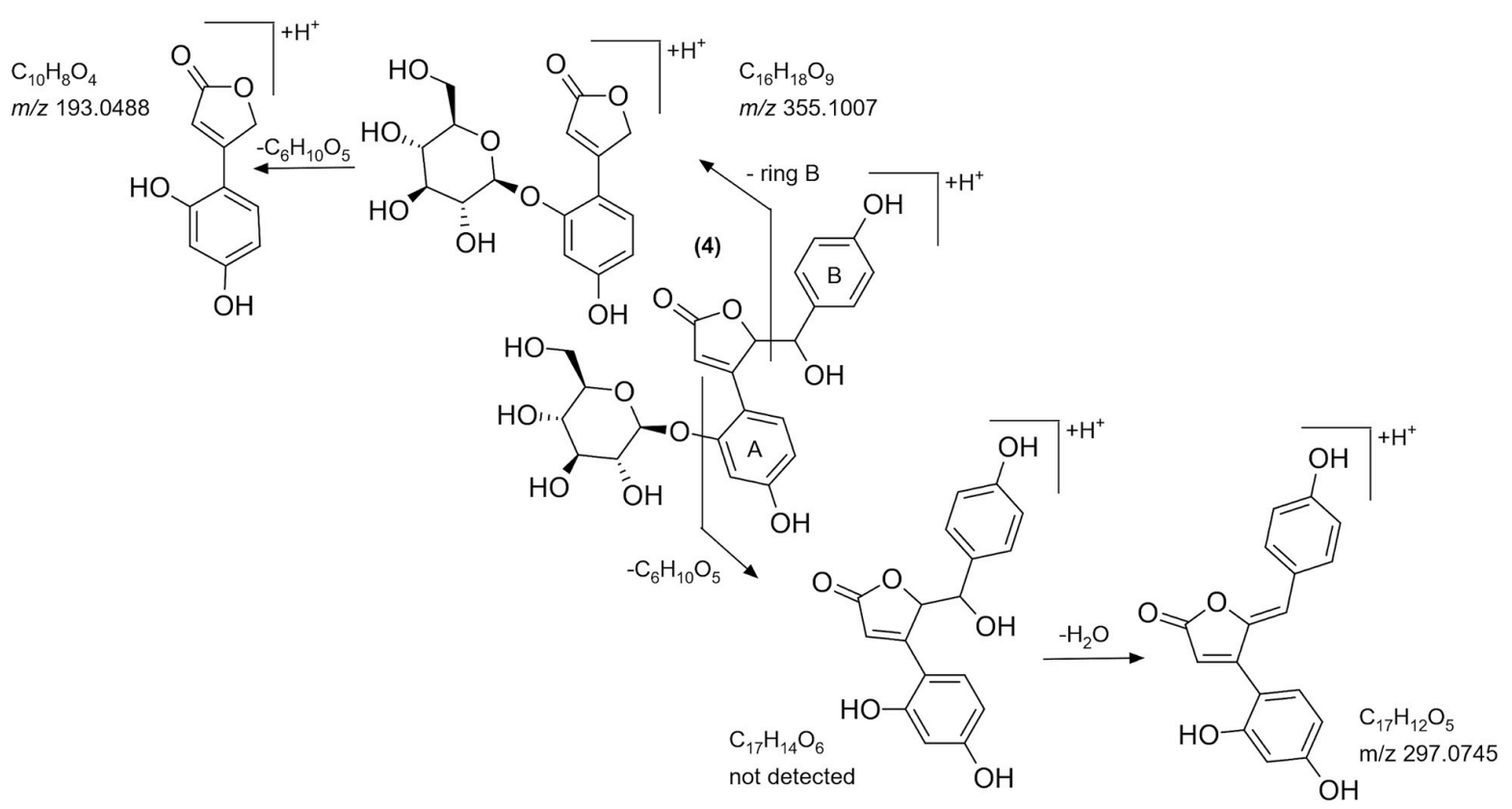

FIGURE 9 | The tentative MS/MS fragmentation pathway of bulatlactone 2"-O- $\beta$-D-glucoside. 


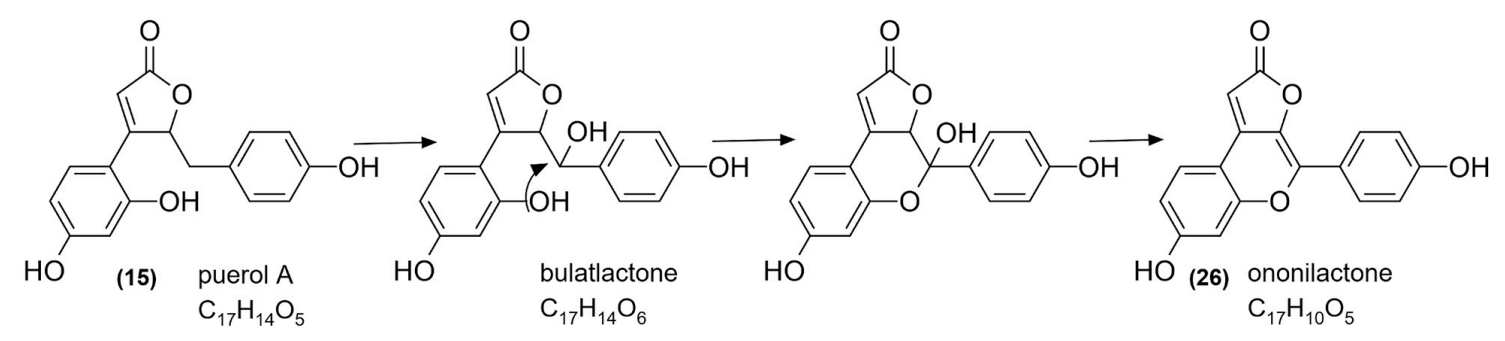

FIGURE 10 | The putative synthetic pathway of ononilactone.

is mentioned in the literature, as a glycosidic compound. Furthermore, scopolin and its aglycone scopoletin were isolated from O. arvensis (Sichinava et al., 2014). Nevertheless, the UV spectrum of these compounds shows an absorption maximum at higher values (Pina et al., 2019), and the fragmentation pathway does not match with that of coumarins (Yi et al., 2014). As a consequence, the isolation of the compound was inevitable to elucidate its structure by NMR spectroscopy. The number of carbon and hydrogen resonances were not in agreement with that of the hypothesized one by the HR-MS measurements $\left(\mathrm{C}_{16} \mathrm{H}_{18} \mathrm{O}_{9}\right)$, but the presence of a glucose unit could be confirmed. The recorded NMR signals showed great similarity with that of puerol A (Ghribi et al., 2015), except for the lack of a $\mathrm{CH}_{2}$ signal at 4 a position. The downfield shifts of $\mathrm{H}-4 \mathrm{a}$ and $\mathrm{C}-4 \mathrm{a}$ indicated the presence of a hydroxy group in geminal position (Figure 5). Regarding the structure of the aglycone and the glucoside drawn from the NMR studies, the molecular formulas $\mathrm{C}_{17} \mathrm{H}_{14} \mathrm{O}_{6}$ and $\mathrm{C}_{23} \mathrm{H}_{24} \mathrm{O}_{11}$ could be calculated, resulting in calculated protonated quasi-molecular masses of 315.0863 and 477.1391. Revising the HR-MS spectrum of peak 4, none of these signals could be detected; however, the $[\mathrm{M}+\mathrm{Na}]^{+},[2 \mathrm{M}+\mathrm{H}]^{+}$, and $[2 \mathrm{M}+\mathrm{Na}]^{+}$ions were present at $m / z$ 499.1224, 953.7456, and 975.6811 , respectively. Instead of the aglycone as a product ion, the $[\mathrm{M}+\mathrm{H} \text {-ring } \mathrm{B}]^{+}$ion could be detected at $\mathrm{m} / z$ 355.1054, which indeed could lose a glucose moiety resulting in the peak at $\mathrm{m} / z 193.0491$ (Figure 9). Neither the aglycone nor the glucoside form of this compound have been described before in the plant kingdom; thus, it is named as bulatlactone $2^{\prime \prime}-O-\beta-D$-glucoside.

The presence and structure of bulatlactone is fascinating from the point of view that it can serve as an intermediate between the well-known puerol derivatives and the newly described structure, ononilactone (Figure 10). As we assume, through a dehydration and a ring closure step, ononilactone could be formed from bulatlactone aglycone. Bulatlactone was only detected in the form of $2^{\prime \prime}$-O-glucoside and not as an aglycone. As the glucosidation takes place through the $2^{\prime \prime}$ hydroxy group (which is involved in the ring closure, as well), it prevents the transformation of bulatlactone to ononilactone and stabilizes this form.

\section{Qualitative Characterization of Phytochemical Composition of Ononis HRCs}

Investigating the isoflavonoid pattern of the HRCs of the species, a reduced spectrum could be observed compared to the native plants. Formononetin, 2'-methoxyformononetin, sativanone, and medicarpin beside their derivatives could be found in both wild-grown (Ghribi et al., 2015) and genetically modified transformed samples abundantly. On the contrary, isoflavonoid derivatives with various skeletons (isoflavone, isoflavanone, and pterocarpan) but with a common methylenedioxy substituent (pseudobaptigenin, cuneatin, onogenin, and maackiain) could only be detected in the HRCs in trace quantities or not at all. Interestingly, pseudobaptigenin derivatives could be observed only in trace quantities in the samples for qualitative analysis; however, in the quantitative samples, their concentration was comparable with formononetin. This could be the consequence of that after lyophilization, quantitative samples were immediately measured, while the qualitative samples were kept airtight and analyzed only weeks after the harvest. The decomposition of pseudobaptigenin in aqueous medium was experienced by our research group during in vitro tests, but in this case, the HRC were kept sealed in a dry form. Moreover, in the qualitative samples, onogenin and maackiain derivatives could be observed, while in quantitative samples, their amounts were under limit of detection. Based on these observations, it is hypothesized that pseudobaptigenin is transformed to onogenin and maackiain. These results show that the measured isoflavonoid content of wild grown samples (Gampe et al., 2020) does not necessarily reflect the isoflavonoid content of the living plant, as it changes with time after harvest. As different isoflavonoids can possess distinct biological effects, the age and the storage conditions can affect the medicinal value of the sample.

\section{Proliferation of HRCs and Comparison of Biomass and Phenolic Compound Accumulation}

The overall isoflavonoid yield of HRCs showed a somewhat higher level, than the wild-grown samples of O. spinosa, and was comparable of that of $O$. arvensis. However, the aim of this study was not to optimize the proliferation and isoflavonoid extractability, thus modifying the circumstances of cultivation can lead to better results. Moreover, the isoflavonoid profile of wild-grown samples markedly differed from the fresh and older samples, too (Gampe et al., 2020). The most characteristic compound produced by the HRCs was medicarpin glucoside $(2.23-2.89 \mathrm{mg} / 100 \mathrm{mg}$ in O. spinosa and $1.69-1.87 \mathrm{mg} / 100 \mathrm{mg}$ in $O$. arvensis), followed by sativanone 
glucoside (0.56-1.14 mg/100 $\mathrm{mg}$ in O. spinosa and 0.13$0.20 \mathrm{mg} / 100 \mathrm{mg}$ in $O$. arvensis) and pseudobaptigenin glucoside (0.12-0.20 mg/100 mg in O. spinosa and 0.09-0.16 mg/100 mg in $O$. arvensis) (Supplementary Tables 2,3 ). In the wild-grown samples, the amount of methoxy and methylenedioxy derivatives are comparable, whereas in HRCs, the methoxy derivatives are the predominant, except for pseudobaptigenin. The observed decrease in the amount of the methylenedioxy compounds could be a result of the genetic modification by the Ri plasmid or the lack of some biotic or abiotic factors that could not be reproduced under in vitro circumstances, e.g., symbiotic Rhizobium strains and drought stress. Regarding the relative isoflavonoid content that decreased from the 1st (O. spinosa) or the 2nd week (O. spinosa), but the fresh and dry weight increased until the 3rd week, it can be assumed that the cultures use their sources mainly for growth and not accumulating specialized metabolites. If the aim is the isolation of isoflavonoid compounds, the 3rd week is optimal, since the absolute quantity of the isoflavonoids was the highest in those days. Usually, the aglycone forms are regarded as the biologically active forms, but unfortunately, fresh HRCs accumulate mainly glycosides. On the other hand, regarding the qualitative studies, upon storage, these can transform to aglycones or the intestinal flora can hydrolyze them to their aglycone form, too.

In conclusion, Ononis hairy root cultures contain some special phenolic lactones beside isoflavonoids. O. spinosa can serve as rich sources of methoxylated isoflavonoids, as it produced them in higher quantities compared to wild-grown plants. Considering that most isoflavonoids with methylenedioxy substituent are missing, the isoflavonoid spectrum of HRCs is less complicated, providing an easy possibility to realize the isolation of the present compounds.

\section{DATA AVAILABILITY STATEMENT}

The original contributions presented in the study are included in the article/Supplementary Material, further inquiries can be directed to the corresponding author.

\section{AUTHOR CONTRIBUTIONS}

ZS recorded and analyzed the NMR spectra for ononilactone and $\mathrm{AD}$ recorded the ones for bulatlactone. $\mathrm{IB}$ recorded the

\section{REFERENCES}

Addotey, J. N., Lengers, I., Jose, J., Gampe, N., Béni, S., Petereit, F., et al. (2018). Isoflavonoids with inhibiting effects on human hyaluronidase-1 and norneolignan clitorienolactone B from Ononis spinosa L. root extract. Fitoterapia 130, 169-174. doi: 10.1016/j.fitote.2018.08.013

Atanasov, A. G., Waltenberger, B., Pferschy-Wenzig, E.-M., Linder, T., Wawrosch, C., Uhrin, P., et al. (2015). Discovery and resupply of pharmacologically active plant-derived natural products: a review. Biotechnol. Adv. 33, 1582-1614. doi: 10.1016/j.biotechadv.2015.08.001

Bertóti, R., Böszörményi, A., Alberti, A., Béni, S., M-Hamvas, M., Szőke, É, et al. (2019). Variability of bioactive glucosinolates, isothiocyanates and enzyme
Orbitrap-MS chromatograms. IK and ÉS started and maintained the hairy root cultures. LK and SB contributed to the design of the experiments and assisted in the interpretation of the data. NG performed all other experiments, analyzed and interpreted the data, and drafted the manuscript with the assistance of SB. All authors read and approved the final version of the manuscript.

\section{FUNDING}

The financial support from the Bolyai fellowship for SB and IB and the support of EFOP-3.6.3-VEKOP-16-201700009 for NG are gratefully acknowledged. This work was supported by the UNNKP-18-3-III-SE-30 New National Excellence Program of the Ministry of Human Capacities, by the Bolyai+ UNKP-20-5-SE-31 New National Excellence Program of the Ministry of Human Capacities, by the National Research Development and Innovation Office (projects: VEKOP-2.3.315-2017-00020, OTKA 135712), and by the ELTE Institutional Excellence Program (1783-3/2018/FEKUTSRAT) supported by the Hungarian Ministry of Human Capacities. The authors would like to acknowledge the financial support from the Central Library of Semmelweis University supporting the Open Access publication.

\section{ACKNOWLEDGMENTS}

NG gratefully acknowledges the help of Andrea Nagyné Nedves and Tamás Czeglédi for their valuable help in sample preparation, Katalin Koller Béláné for managing the in vitro cultures, Rita Könye and Regina Bertóti-Gond for their help in the PCR studies, and Máté Kemecsei for naming the new structures. The authors thank Prof. Csaba Szántay Jr. for supporting this project by NMR spectrometer time at Gedeon Richter Plc.

\section{SUPPLEMENTARY MATERIAL}

The Supplementary Material for this article can be found online at: https://www.frontiersin.org/articles/10.3389/fpls.2020. 622585/full\#supplementary-material

patterns in horseradish hairy root cultures initiated from different organs. Molecules 24:2828. doi: 10.3390/molecules24152828

Clifford, M., and Brown, J. E. (2006). "Dietary flavonoids and health - broadening the perspective," in Flavonoids: Chemistry, Biochemistry and Applications, eds O. M. Andersen and K. R. Markham (Boca Raton, FL: CRC Press), $321-332$.

Cuyckens, F., and Claeys, M. (2004). Mass spectrometry in the structural analysis of flavonoids. J. Mass Spectrom. 39, 1-15. doi: 10.1002/jms. 585

Davies, K. M., and Schwinn, K. E. (2006). "Molecular biology and biotechnology of flavonoid biosynthesis," in Flavonoids: Chemistry, Biochemistry and Applications, eds O. Andersen and K. Markham (Boca Raton, FL: CRC Press), 143-217. doi: 10.1201/9781420039443.ch3 
de Rijke, E., de Kanter, F., Ariese, F., Brinkman, U. A. Th, and Gooijer, C. (2004). Liquid chromatography coupled to nuclear magnetic resonance spectroscopy for the identification of isoflavone glucoside malonates in T. pratense L. leaves. J. Sep. Sci. 27, 1061-1070. doi: 10.1002/jssc.200401844

Deipenbrock, M., Sendker, J., and Hensel, A. (2020). Aqueous root extract from Ononis spinosa exerts anti-adhesive activity against Uropathogenic Escherichia coli. Planta Med. 86, 247-254. doi: 10.1055/a-1089-8645

Farag, M. A., Huhman, D. V., Lei, Z., and Sumner, L. W. (2007). Metabolic profiling and systematic identification of flavonoids and isoflavonoids in roots and cell suspension cultures of Medicago truncatula using HPLC-UV-ESI-MS and GC-MS. Phytochemistry 68, 342-354. doi: 10.1016/j.phytochem.2006.10.023

Furner, I. J., Huffman, G. A., Amasino, R. M., Garfinkel, D. J., Gordon, M. P., and Nester, E. W. (1986). An Agrobacterium transformation in the evolution of the genus Nicotiana. Nature 319, 422-427. doi: 10.1038/319422a0

Gamborg, O. L., Miller, R. A., and Ojima, K. (1968). Nutrient requirements of suspension cultures of soybean root cells. Exp. Cell Res. 50, 151-158. doi: 10. 1016/0014-4827(68)90403-5

Gampe, N., Darcsi, A., Kursinszki, L., and Béni, S. (2018a). Separation and characterization of homopipecolic acid isoflavonoid ester derivatives isolated from Ononis spinosa L. root. J. Chromatogr. B Analyt. Technol. Biomed. Life Sci. 1091, 21-28. doi: 10.1016/j.jchromb.2018.05.023

Gampe, N., Darcsi, A., Lohner, S., Béni, S., and Kursinszki, L. (2016). Characterization and identification of isoflavonoid glycosides in the root of Spiny restharrow (Ononis spinosa L.) by HPLC-QTOF-MS, HPLC-MS/MS and NMR. J. Pharm. Biomed. Anal. 123, 74-81. doi: 10.1016/j.jpba.2016.01.058

Gampe, N., Darcsi, A., Nagyné Nedves, A., Boldizsár, I., Kursinszki, L., and Béni, S. (2018b). Phytochemical analysis of Ononis arvensis L. by liquid chromatography coupled with mass spectrometry. J. Mass Spectrom. 54, 121133. doi: $10.1002 /$ jms. 4308

Gampe, N., Nagy, E., Kursinszki, L., and Béni, S. (2020). Quantitative determination of isoflavonoids in Ononis species by UPLC-UV-DAD. Phytochem. Anal. doi: 10.1002/PCA.2995. [Epub ahead of print].

Georgiev, M. I., Agostini, E., Ludwig-Müller, J., and Xu, J. (2012). Genetically transformed roots: from plant disease to biotechnological resource. Trends Biotechnol. 30, 528-537. doi: 10.1016/j.tibtech.2012.07.001

Ghribi, L., Waffo-Téguo, P., Cluzet, S., Marchal, A., Marques, J., Mérillon, J.-M. M., et al. (2015). Isolation and structure elucidation of bioactive compounds from the roots of the Tunisian Ononis angustissima L. Bioorg. Med. Chem. Lett. 25, 3825-3830. doi: 10.1016/j.bmcl.2015.07.076

Kuzovkina, I. N., Mantrova, O. V., Al'terman, I. E., and Yakimov, S. A. (1996). Culture of genetically transformed hairy roots derived from anthraquinoneproducing European madder plants. Russ. J. Plant Physiol. 43, 252-258.

Larsson, J., Gottfries, J., Bohlin, L., and Backlund, A. (2005). Expanding the ChemGPS chemical space with natural products. J. Nat. Prod. 68, 985-991. doi: $10.1021 / \mathrm{np} 049655 \mathrm{u}$

Markham, K. R., and Mabry, T. J. (1975). "Ultraviolet-visible and proton magnetic resonance spectroscopy of flavonoids," in The Flavonoids, ed. J. B. Harborne (Boston, MA: Springer), 45-77. doi: 10.1007/978-1-4899-2909-9_2

Metabocard of Chlorogenic acid (HMDB0003164) (2006). The Human Metabolom Database. Available online at: http://www.hmdb.ca/metabolites/ HMDB0003164\#spectra (accessed July 15, 2019).

Murashige, T., and Skoog, F. (1962). A revised medium for rapid growth and bio assays with tobacco tissue cultures. Physiol. Plant. 15, 473-497. doi: 10.1111/j. 1399-3054.1962.tb08052.x

Paßreiter, C. M. (1992). Co-occurrence of 2-pyrrolidineacetic acid with the pyrrolizidines tussilaginic acid and isotussilaginic acid and their 1-epimers in
Arnica species and Tussilago farfara. Phytochemistry 31, 4135-4137. doi: 10. 1016/0031-9422(92)80428-H

Pina, J., de Castro, C. S., Delgado-Pinar, E., and Sérgio Seixas de Melo, J. (2019). Characterization of 4-methylesculetin and of its mono- and dimethoxylated derivatives in water and organic solvents in its ground, singlet and triplet excited states. J. Mol. Liq. 278, 616-626. doi: 10.1016/j.molliq.2019. 01.083

Sichinava, M. B., Mchelidze, K. Z., Churadze, M. V., Alaniia, M. D., and Aneli, D. N. (2014). Chemical composition and microstructural peculiarities of overground and underground vegetative organs of field restharrow (Ononis arvensis L.). Georgian Med. News 231, 88-94.

Spiegler, V., Gierlikowska, B., Saenger, T., Addotey, J. N., Sendker, J., Jose, J., et al. (2020). Root extracts from Ononis spinosa inhibit IL-8 release via interactions with toll-like receptor 4 and lipopolysaccharide. Front. Pharmacol. 11:889. doi: 10.3389/fphar.2020.00889

Stojković, D., Dias, M. I, Drakulić, D., Barros, L., Stevanović, M., C. F. R. Ferreira, I., et al. (2020). Methanolic extract of the herb Ononis spinosa L. is an antifungal agent with no cytotoxicity to primary human cells. Pharmaceuticals 13:78. doi: 10.3390/ph13040078

Stojkovic, D., Drakulic, D., Gašic, U., Zengin, G., Stevanovic, M., Rajcevic, N., et al. (2020). Ononis spinosa L., an edible and medicinal plant: UHPLC-LTQ-Orbitrap/MS chemical profiling and biological activities of the herbal extract. Food Funct. 11, 7138-7151. doi: 10.1039/d0fo0 $1595 d$

Tůmová, L., Bartáková, M., and Zabloudilova, J. (2003). Iodoacetic acid as a potential elicitor of increased formation of flavonoids in the culture of Ononis arvensis L. in vitro. Ceská. Slov. Farm. 52, 189-192.

Tůmová, L., and Polívková, D. (2006). Effect of AgNO3 on the production of flavonoids by the culture of Ononis arvensis L. in vitro. Ceska. Slov. Farm. 55, $186-188$.

Tůmová, L., and Rusková, R. (1998). Effect of CdCl2 and CuSO4 on the production of flavonoids by the culture of Ononis arvensis L. in vitro. Ceská. Slov. Farm. 47, 261-263.

Tůmová, L., Tůma, J., and Dolezal, M. (2011). Pyrazinecarboxamides as potential elicitors of flavonolignan and flavonoid production in Silybum marianum and Ononis arvensis cultures in vitro. Molecules 16, 9142-9152. doi: 10.3390/ molecules 16119142

Yi, T., Zhu, L., Tang, Y.-N., Zhang, J.-Y., Liang, Z.-T., Xu, J., et al. (2014). An integrated strategy based on UPLC-DAD-QTOF-MS for metabolism and pharmacokinetic studies of herbal medicines: Tibetan "Snow Lotus" herb (Saussurea laniceps), a case study. J. Ethnopharmacol. 153, 701-713. doi: 10. 1016/j.jep.2014.03.031

Conflict of Interest: ZS was employed by the company Gedeon Richter Plc.

The remaining authors declare that the research was conducted in the absence of any commercial or financial relationships that could be construed as a potential conflict of interest.

Copyright (c) 2021 Gampe, Szakács, Darcsi, Boldizsár, Szőke, Kuzovkina, Kursinszki and Béni. This is an open-access article distributed under the terms of the Creative Commons Attribution License (CC BY). The use, distribution or reproduction in other forums is permitted, provided the original author(s) and the copyright owner(s) are credited and that the original publication in this journal is cited, in accordance with accepted academic practice. No use, distribution or reproduction is permitted which does not comply with these terms. 